\title{
Transfert des bactéries fécales vers une nappe phréatique à travers une colonne de sol en région équatoriale : influence de la charge en eau appliquée en surface
}

\section{Faecal bacteria transfer through a soil column to the groundwater in an equatorial region: influence of the applied surface water load}

\author{
M. Nola, T. Njiné, N. Kemka, S.H. Zébazé Togouet, P. Servais, M. Messouli, Cl. \\ Boutin, A. Monkiedje et S. Foto Menbohan
}

Volume 19, numéro 2, 2006

URI : https://id.erudit.org/iderudit/013044ar

DOI : https://doi.org/10.7202/013044ar

Aller au sommaire du numéro

Éditeur(s)

Université du Québec - INRS-Eau, Terre et Environnement (INRS-ETE)

ISSN

1718-8598 (numérique)

Découvrir la revue

Citer cet article

Nola, M., Njiné, T., Kemka, N., Togouet, S. Z., Servais, P., Messouli, M., Boutin, C., Monkiedje, A. \& Menbohan, S. F. (2006). Transfert des bactéries fécales vers une nappe phréatique à travers une colonne de sol en région équatoriale : influence de la charge en eau appliquée en surface. Revue des sciences de l'eau / Journal of Water Science, 19(2), 101-112. https://doi.org/10.7202/013044ar
Résumé de l'article

L'alimentation des eaux souterraines procède entre autres par des apports d'eau d'infiltration à travers les différents horizons du sol qui séparent la surface du sol du toit de la nappe phréatique. Une étude a été réalisée au laboratoire sur le rôle de la charge d'eau introduite dans une colonne de sol de $1 \mathrm{~m}$ de hauteur située au toit de la nappe, dans le transfert à l'eau souterraine, des bactéries indicatrices de pollution de l'eau de boisson. Les charges d'eaux usées de $50 \mathrm{~mL}, 100 \mathrm{~mL}$ et $250 \mathrm{~mL}$ ont été appliquées. Les analyses des eaux avant et après percolation ont concerné les coliformes thermotolérants et streptocoques fécaux, pour les paramètres bactériologiques, et le $\mathrm{pH}, \mathrm{NH}_{4}^{+}$et la conductivité électrique, pour les paramètres chimiques.

Les résultats révèlent une réduction du nombre de microorganismes dans les eaux qui ont percolé à travers la colonne du sol. Cette réduction est imputable à la rétention de ces cellules par la colonne de sol. À la charge de $50 \mathrm{~mL}$ d'eau appliquée au-dessus de la colonne du sol, cette réduction a été de l'ordre de 7 unités logarithmiques pour les coliformes thermotolérants, et de 6 pour les streptocoques fécaux. En appliquant la charge de $250 \mathrm{~mL}$, la réduction a plutôt été de l'ordre de 6 unités logarithmiques pour les coliformes thermotolérants, et de 7 pour les streptocoques fécaux. Cette réduction de la concentration microbienne circulante observée dans les eaux percolées a été de l'ordre de 7 unités logarithmiques pour les deux groupes de bactéries lorsque la charge de $100 \mathrm{~mL}$ a été appliquée. À charge d'eau usée élevée, la colonne de sol semble ainsi retenir plus de streptocoques fécaux que de coliformes thermotolérants. Ce comportement de la colonne de sol semble s'inverser lorsque la charge d'eau appliquée est relativement faible. Les éléments chimiques sont également retenus par le sol. Les caractéristiques des eaux qui percolent évoluent dans le temps, montrant que la rétention des polluants des eaux d'infiltration par une colonne de sol est un processus dynamique.
Ce document est protégé par la loi sur le droit d'auteur. L’utilisation des services d’Érudit (y compris la reproduction) est assujettie à sa politique d'utilisation que vous pouvez consulter en ligne.

https://apropos.erudit.org/fr/usagers/politique-dutilisation/ 


\title{
TRANSFERT DES BACTÉRIES FÉCALES VERS UNE NAPPE PHRÉATIQUE À TRAVERS UNE COLONNE DE SOL EN RÉGION ÉQUATORIALE : INFLUENCE DE LA CHARGE EN EAU APPLIQUÉE EN SURFACE
}

\author{
Faecal bacteria transfer through a soil column to the groundwater \\ in an equatorial region: influence of the applied surface water load
}

\section{Nola ${ }^{1 *}$ T. Njinét, N. Kemka ${ }^{1,2}$, S.H. Zébazé Togouet', P. Servais', M. Messoulit, Cl. Boutin ${ }^{5}$, A. Monkiedje', S. Foto Menbohan ${ }^{1}$}

\begin{abstract}
${ }^{1}$ Université de Yaoundé I, Faculté des Sciences, Laboratoire de Biologie Générale, B.P. 812 Yaoundé, Cameroun.
${ }^{2}$ Institut de Recherches Géologiques et Minières, Centre de Recherches Hydrologiques, B.P. 4110 Nlongkak -Yaoundé, Cameroun.

${ }^{3}$ Université Libre de Bruxelles, Laboratoire d'Écologie des Systèmes Aquatiques, Campus de la Plaine, CP 221, Boulevard du Triomphe, 1050 Bruxelles, Belgique.

${ }^{4}$ Université Cadi Ayyad, Faculté des Sciences Semlalia, Laboratoire d'Hydrobiologie et Écologie Souterraine, B.P. 2390 Bd Moulay Abdellah, Marrakech, Maroc.

${ }^{5}$ Université Paul Sabatier CNRS, Laboratoire de Dynamique de la Biodiversité, UMR 5172, 118 Route de Narbonne, Bât. 4, R3, 31062 Toulouse Cedex 4, France.
\end{abstract}

Reçu le 3 janvier 2005, accepté le 17 juin 2005

\section{SUMMARY}

Water percolation through different soil horizons is one of the main mechanisms contributing to the improvement of the microbial quality of ground water. These soil horizons separate the soil surface from the groundwater table. Wastewater often contains chemicals and microbial pollutants, generally at high concentrations. On the other hand, ground water constitutes a major natural resource in most regions of the world. The present study was carried to examine the transfer of bacterial pollutants to the ground water, with the objective of evaluating the influence of the rate at which wastewater percolates through a soil column overlying the groundwater table.
The soil column was $25 \mathrm{~cm}$ in diameter and one meter high. It was composed of two horizons of different heights with $\mathrm{pH}$ values that varied from 4.43 to 4.56 . Wastewater percolation tests were carried out with volumes of $50 \mathrm{~mL}$, $100 \mathrm{~mL}$ and $250 \mathrm{~mL}$, which were introduced every 30 minutes for each experiment. Chemical analysis was performed for $\mathrm{pH}, \mathrm{NH}_{4}^{+}$and electrical conductivity. Bacteriological analysis was also carried out for thermo-tolerant coliforms and faecal streptococci quantification. These analyses were first carried out on each wastewater sample before introduction into the soil column, and then again after their percolation through the soil column.

Results showed that the lapse of time needed to observe the first percolated water drop was longer for low water loads

*Auteur pour correspondance : 
than for the higher water loading rates. On the other hand, the time necessary to collect an adequate volume of percolated water for analysis was shorter for low water loads than for the high water loads. The average volume of percolated water per hour was thus high at low water loads, and relatively low at high water loading rates.

A comparison of the microbial characteristics of the introduced wastewater and those of the percolated water showed that the bacterial load in the percolated water was lower. This reduction was due to bacterial retention by the soil column. At an applied load of $50 \mathrm{~mL}$, this reduction was of the order of $7 \mathrm{log}$ units for thermo-tolerant coliforms, and $6 \log$ units for faecal streptococci. When water load of $250 \mathrm{~mL}$ was applied, the reduction was of the order of $6 \mathrm{log}$ units for thermo-tolerant coliforms, and $7 \log$ units for faecal streptococci. The reduction was in order of $7 \log$ units for both bacterial groups at an applied wastewater loading of $100 \mathrm{~mL}$. It thus appears that, at high applied wastewater loadings, the soil column retained faecal streptococci better than thermotolerant coliforms. The soil column behaviour was reversed when low wastewater loads were applied. It was also noted that for electrical conductivity, a reduction varying from 6,240 to $6,550 \mu \mathrm{S} / \mathrm{cm}$ was obtained in the water leaving the soil column. The concentration of ammonia decreased from $44-50 \mathrm{mg} / \mathrm{L}$ at the entrance to the column to around $1 \mathrm{mg} / \mathrm{L}$ at the exit of the column. Average $\mathrm{pH}$ values of water percolated through the two soil horizons varied from 5.70 to 7.32 , whereas $\mathrm{pH}$ values of water introduced into the soil column varied from 7.43 to 8.02. It thus appears that the $\mathrm{pH}$ of these two soil horizons strongly influenced the $\mathrm{pH}$ of the percolating water that would enter the underlying ground water. The chemical and bacteriological characteristics of percolating water exhibited temporal variations, showing that the retention of pollutants by the soil column was a dynamic process.

Key words: thermo-tolerant coliforms, faecal streptococci, water percolation, soil column, water loading, ground water.

\section{RÉSUMÉ}

L'alimentation des eaux souterraines procède entre autres par des apports d'eau d'infiltration à travers les différents horizons du sol qui séparent la surface du sol du toit de la nappe phréatique. Une étude a été réalisée au laboratoire sur le rôle de la charge d'eau introduite dans une colonne de sol de $1 \mathrm{~m}$ de hauteur située au toit de la nappe, dans le transfert à l'eau souterraine, des bactéries indicatrices de pollution de l'eau de boisson. Les charges d'eaux usées de $50 \mathrm{~mL}, 100 \mathrm{~mL}$ et $250 \mathrm{~mL}$ ont été appliquées. Les analyses des eaux avant et après percolation ont concerné les coliformes thermotolérants et streptocoques fécaux, pour les paramètres bactériologiques, et le $\mathrm{pH}, \mathrm{NH}_{4}^{+}$et la conductivité électrique, pour les paramètres chimiques.

Les résultats révèlent une réduction $\mathrm{du}$ nombre de microorganismes dans les eaux qui ont percolé à travers la colonne du sol. Cette réduction est imputable à la rétention de ces cellules par la colonne de sol. À la charge de $50 \mathrm{~mL}$ d'eau appliquée au-dessus de la colonne du sol, cette réduction a été de l'ordre de 7 unités logarithmiques pour les coliformes thermotolérants, et de 6 pour les streptocoques fécaux. En appliquant la charge de $250 \mathrm{~mL}$, la réduction a plutôt été de l'ordre de 6 unités logarithmiques pour les coliformes thermotolérants, et de 7 pour les streptocoques fécaux. Cette réduction de la concentration microbienne circulante observée dans les eaux percolées a été de l'ordre de 7 unités logarithmiques pour les deux groupes de bactéries lorsque la charge de $100 \mathrm{~mL}$ a été appliquée. À charge d'eau usée élevée, la colonne de sol semble ainsi retenir plus de streptocoques fécaux que de coliformes thermotolérants. Ce comportement de la colonne de sol semble s'inverser lorsque la charge d'eau appliquée est relativement faible. Les éléments chimiques sont également retenus par le sol. Les caractéristiques des eaux qui percolent évoluent dans le temps, montrant que la rétention des polluants des eaux d'infiltration par une colonne de sol est un processus dynamique.

\section{Mots clés : coliformes thermotolérants, streptocoques fécaux, charge d'eau, colonne du sol, eau d'infiltration, nappe phré- atique.}

\section{INTRODUCTION}

L'alimentation des eaux souterraines procède par des infiltrations verticales des eaux de ruissellement issues des précipitations, et des infiltrations horizontales des eaux de surface. Les risques de leur pollution chimique et biologique dépendent d'une part des caractéristiques des eaux d'infiltration, et d'autre part, des propriétés naturelles des couches géologiques qui séparent la nappe phréatique de la surface du sol.

L'origine des bactéries dans divers types d'eaux souterraines est encore discutée actuellement. Bien que pour certains auteurs l'origine d'autres microorganismes soit incertaine (MAYER et al., 1997; DZEDA et al., 1998), la plupart des bactéries des eaux souterraines proviennent du milieu de surface par transport lors de l'infiltration des eaux de surface vers les eaux souterraines (MADSEN et GHIORSE, 1993; STEVENS, 1997). Pour FENCHEL (2001), les ancêtres des bactéries autochtones dans les écosystèmes aquatiques souterrains proviendraient des microorganismes anoxigéniques qui auraient évolué. Selon VAN ELSAS et HEIJNEN (1990), des conditions optimales de survie sont indispensables pour 
l'introduction du microorganisme bactérien dans le sol, et son transfert à la nappe phréatique.

L'abondance des bactéries dans l'eau souterraine est à la fois tributaire de la température et de la perméabilité du sol, et des conditions physico-chimiques et trophiques locales, bien que certaines espèces bactériennes préfèrent des biotopes oligotrophes (GOUNOT, 1994; HOLT et al., 2000). Les qualités physico-chimique et microbiologique des eaux souterraines subissent parfois des variations spatiotemporelles, dépendant souvent de facteurs météorologiques et pédologiques. La qualité physico-chimique de l'eau évolue généralement en relation avec l'évolution naturelle et temporelle des sols encaissants (KORKKA-NIEMI et LAIKARI, 1994; HELENA et al., 2000). L'infiltration, la persistance, la survie et l'évolution des micro-organismes présents sont liées à tout un ensemble de facteurs comprenant notamment la nature du sol, les conditions environnementales prévalant dans ce sol et dans l'eau souterraine, et la qualité des eaux superficielles infiltrées (TEUSTCH et al., 1991; FENCHEL, 2001).

Dans la majorité des métropoles des pays en développement, les eaux usées domestiques sont déversées directement dans la nature sans aucun traitement préalable, suite à l'absence ou au dysfonctionnement des stations d'épuration existantes. Ces eaux sont susceptibles de polluer le sol et le sous-sol, et par la suite les eaux souterraines, en raison de leurs teneurs souvent élevées en matières organiques biodégradables et en microorganismes d'origine fécale qui peuvent atteindre jusqu'à $6 \times 10^{7}$ UFC/100 mL (NJINE et al., 2001). De plus, le développement accéléré et incontrôlé des villes et la multiplication des quartiers dépourvus de tout plan d'urbanisation entraînent une prolifération des systèmes individuels d'assainissement inadéquats (LE JALLE, 1998).

En région équatoriale au Cameroun, les eaux souterraines sont acides, douces, faiblement minéralisées, et hébergent une microflore variée (NOLA et al., 1998). Les niveaux piézométriques des eaux souterraines et les profondeurs des puits influencent la distribution de cette microflore (NOLA et al., 2000). Dans ces eaux, les valeurs des éléments chimiques tels le $\mathrm{pH}$, la conductivité électrique, les teneurs en ions calcium, magnésium, sodium, potassium, et les concentrations en $\mathrm{CO}_{2}$ affectent à des degrés divers, la distribution des bactéries témoins de contamination fécale et de certaines bactéries pathogènes opportunistes telles Pseudomonas aeruginosa et Aeromonas hydrophila (NOLA et al., 2001, 2002).

Si quelques informations sont disponibles sur la qualité bactériologique des eaux souterraines de cette région équatoriale au Cameroun, on sait cependant peu de chose sur le rôle que peuvent jouer le sol et le sous-sol dans la propagation des polluants chimiques et microbiologiques, depuis la surface du sol jusqu'à l'eau souterraine. Le présent travail vise à évaluer le comportement de deux groupes de bactéries, les coliformes thermotolérants et les streptocoques fécaux, contenues dans les eaux usées en fonction de la charge appliquée à une colonne de deux horizons de sol directement en contact avec le toit d'une nappe phréatique.

\section{MATÉRIELS ET MÉTHODES}

\subsection{Site d'étude}

La région de Yaoundé (Cameroun) est située à $3^{\circ} 52^{\prime}$ de latitude Nord et $11^{\circ} 32^{\prime}$ de longitude Est, à une altitude moyenne de $759 \mathrm{~m}$. Les formations géologiques sont des roches hétérogènes plus ou moins quartzeuses ou feldspathiques, avec des lits minéraux qui comprennent de la biotite, du grenat, de l'amphibole et du disthène. Le sol est de nature rouge latéritique forestier classique; il est insaturé et a un $\mathrm{pH}$ acide qui est en général inférieur à 5 (BACHELIER, 1959; YONGUE-FOUATEU, 1986). Le pourcentage de sable est plus élevé en surface $(73 \%)$ et diminue au fur et à mesure que la profondeur du sol augmente (HUMBEL, 1969). La porosité et la densité réelles du sol fluctuent respectivement de 5 à $71 \%$ et de 2,7 à 3,1 (BACHELIER, 1959; HUMBEL, 1969). Ce sol de Yaoundé a une perméabilité verticale qui varie de 50 à $300 \mathrm{~cm} / \mathrm{h}$ près de la surface. Elle diminue jusqu'à 0,5 dans l'horizon meuble pour ensuite augmenter dans l'horizon grossier.

\subsection{Propriétés physiques des horizons du sol et confection de la colonne de sol en carotte}

Aux mois de janvier et février 2000 qui correspondent à la grande saison sèche dans la région de Yaoundé, un trou circulaire de $180 \mathrm{~cm}$ de diamètre a été creusé jusqu'au jaillissement de l'eau de la nappe phréatique. Ce trou a une profondeur de $914 \mathrm{~cm}$ et traverse huit horizons différents de sol. Ces horizons ont été désignés $\mathrm{H}_{1}, \mathrm{H}_{2}, \ldots, \mathrm{H}_{8}$. L'horizon $\mathrm{H}_{1}$, le plus superficiel, est en relation avec la surface du sol, et l'horizon $\mathrm{H}_{8}$, le plus profond, est en relation avec le toit de la nappe phréatique. La figure 1 montre la succession des différents horizons de sol, de la surface du sol au toit de la nappe phréatique.

L'horizon $\mathrm{H}_{7}$ a $115 \mathrm{~cm}$ d'épaisseur et est caractérisé par un matériau compact aux taches brun-jaunâtres en augmentation de volume à la base, avec apparition des taches blanchâtres, des grains de quartz fissurés et translucides, et par une structure polyédrique. Il y subsiste des passés isaltériques à la base. L'horizon $\mathrm{H}_{8}$ a $35 \mathrm{~cm}$ d'épaisseur et est caractérisé par des taches blanchâtres et des taches jaunâtres limono-argileuses, des taches rouge violet à brun marron légèrement indurées. Les taches rouges sont situées au cœur des taches jaunes, qui elles sont en contact avec les taches blanchâtres. Il y a présence de 


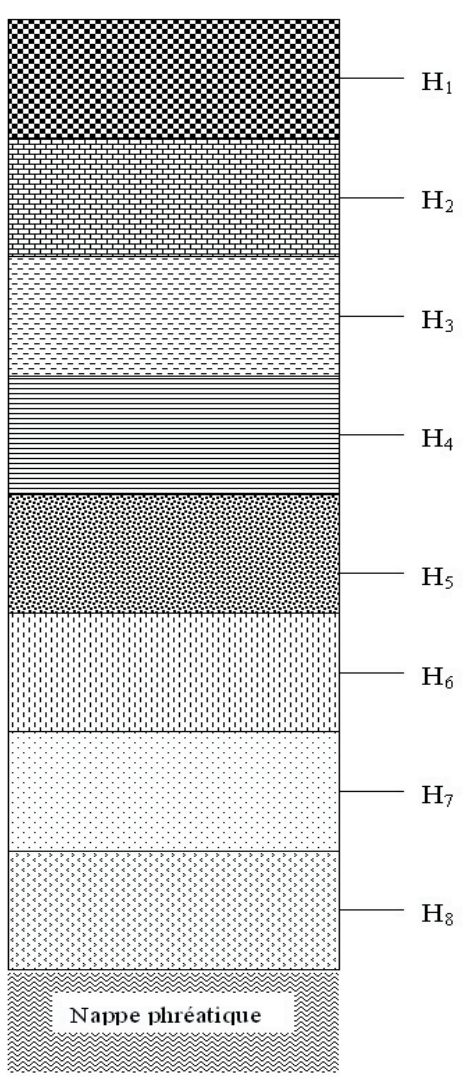

Figure 1 Schéma de la succession des horizons de sol, de la surface du sol au toit de la nappe phréatique (l'épaisseur des différents horizons n'est pas prise en compte sur ce schéma).

Diagram showing soil layer sequence from soil surface to the groundwater table (the thickness of the different soil layers is not considered in this diagram).

grains de quartz et de lits quartzo-feldspathiques. Le matériau est peu compact, et la structure est peu ou pas conservée. La subdivision du sol en différents horizons et la détermination de leurs propriétés macroscopiques ont été basées sur les critères proposés par MARTIN (1967) et MULLER (1977). Les couleurs de chaque horizon ont été déterminées en utilisant le code MUNSEL (Macbeth Division of Kollmorgen Corporation, 1975).

En se servant d'un support pour grimper sur la bordure du puits creusé, une circonscription circulaire de cette bordure a été taraudée de manière à confectionner une colonne cylindrique de sol, semblable à une bougie. Cette colonne de sol, enfouie dans un tuyau PVC, est appelée carotte. Ainsi, après élimination des horizons $\mathrm{H}_{1} \grave{a}_{6} \mathrm{H}_{6}$, une bougie (carotte) de sol de $100 \mathrm{~cm}$ de hauteur et de $25 \mathrm{~cm}$ de diamètre a été confectionnée. Cette carotte est composée de $65 \mathrm{~cm}$ de sol de la partie inférieure de l'horizon $\mathrm{H}_{7}$, et de $35 \mathrm{~cm}$ de sol de l'horizon $\mathrm{H}_{8}$. Pour cette étude, l'horizon $\mathrm{H}_{7}$ sera appelé $\mathrm{H}_{5}$ (formant la couche supérieure de la carotte), et l'horizon $\mathrm{H}_{8}$ sera appelé $\mathrm{H}_{\mathrm{I}}$ (formant la couche inférieure de la carotte). L'extrémité postérieure de cette bougie a ensuite été emballée

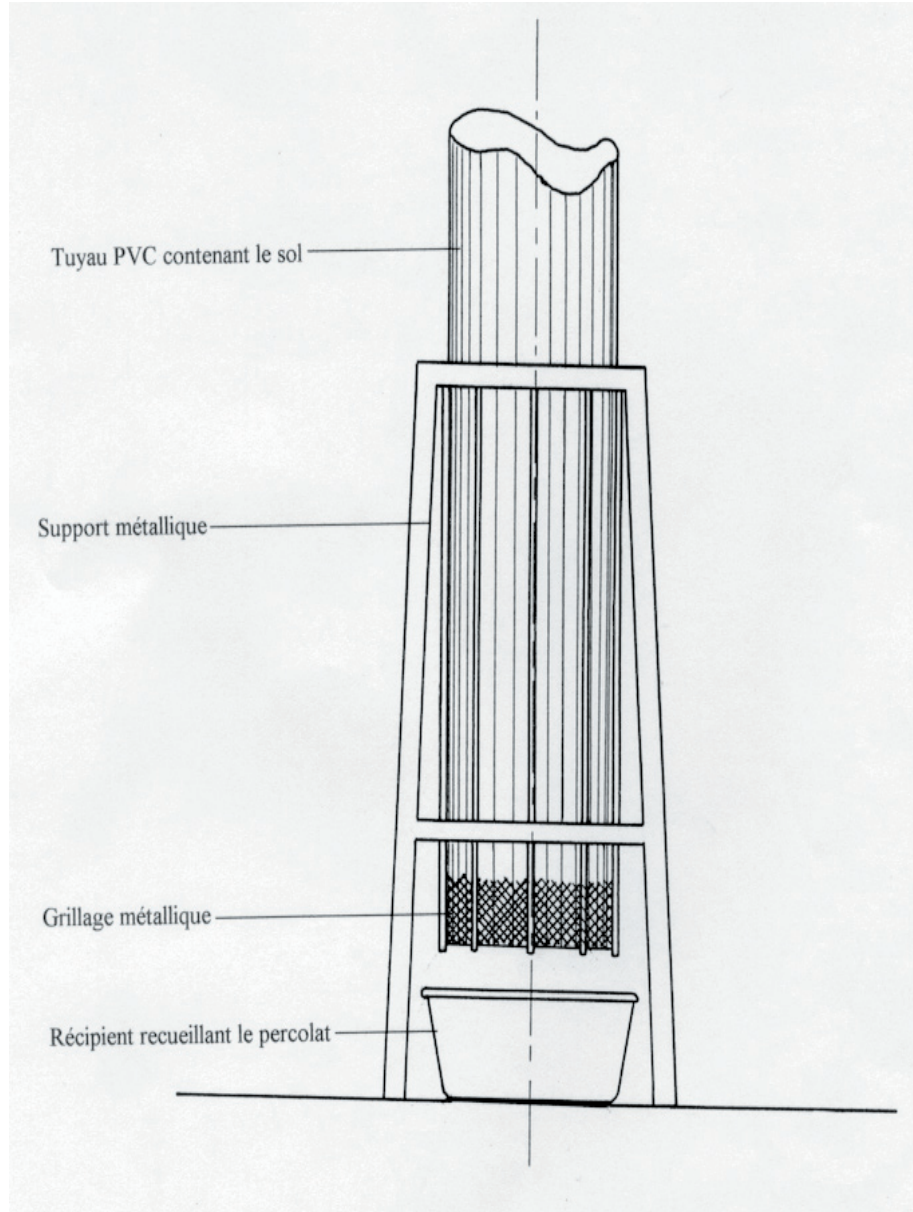

Figure 2 Disposition au laboratoire, de la carotte de sol dans un support métallique.

Laboratory set-up of the soil column in a metal support.

dans un grillage métallique. La bougie a ensuite été transportée au laboratoire et disposée dans un support métallique (Figure 2).

Les deux horizons de sol considérés dans cette étude se distinguent des autres. Il a en effet été noté qu'en présence d'une matière organique biodégradable introduite dans le milieu aqueux, les taux de croissance apparent de Escherichia coli en présence des particules de chacun des deux horizons considérés, pris isolément, sont relativement faibles par rapport aux six autres horizons supérieurs de sol (NOLA et al., 2004a). Il a aussi été indiqué que lors de l'adsorption de Streptococcus faecalis sur les particules isolées des horizons de sol de cette région, les coefficients de linéarité sont relativement faibles en présence des particules de sol de chacun des deux horizons de sol du toit de la nappe d'eau souterraine (NOLA et al., 2004b). Ce coefficient de linéarité est inversement proportionnel à l'intensité de rétention cellulaire (SCHIJVEN et HASSANIZADEH, 2000), et est parfois appelé facteur d'hétérogénéité d'énergie des sites d'adsorption (SERRANO, 2001). 


\subsection{Propriétés chimiques des horizons de la carotte de sol}

Après la confection de la carotte de sol, un échantillon de 2 à $3 \mathrm{~kg}$ de sol de chacun des horizons $\mathrm{H}_{\mathrm{S}}$ et $\mathrm{H}_{\mathrm{I}}$ a été prélevé, et ramené au laboratoire. Les paramètres chimiques analysés ont étéla teneur en fer $(\mathrm{Fe})$, en azote total $(\mathrm{N})$, en phosphore total $(\mathrm{P})$ et en carbone organique $(\mathrm{C})$, le $\mathrm{pH}$, et la capacité de rétention en eau. Après minéralisation des échantillons de sol selon la technique classique de l'attaque triacide (CLAISSE, 1968; NJOPWOUO et ORLIAC, 1979), les analyses chimiques ont été effectuées par les méthodes spectrophotométriques pour le fer, l'azote total et le phosphore total, et par oxydation à chaud au permanganate de potassium en milieu acide sulfurique pour le carbone organique (RODIER, 1996). Le pH de chaque horizon de sol a été mesuré en utilisant une solution de $\mathrm{KCl}$ (1N), suivant la technique proposée par SOIL AND PLANT ANALYSIS COUNCIL (1999). La capacité de rétention en eau a été évaluée en utilisant $15 \mathrm{~g}$ de terre passée au tamis de $2 \mathrm{~mm}$ de diamètre de maille, suivant le protocole proposé par DEMOLON et LEROUX (1952) modifiée.

\subsection{Percolation des eaux et analyse bactériologique}

Après 24 mois de séchage de la carotte de sol à la température du laboratoire $\left(21 \pm 1{ }^{\circ} \mathrm{C}\right)$ dans le but d'éliminer la microflore cultivable, les tests préliminaires de percolation de l'eau distillée stérilisée ont révélé que les eaux percolées ne contenaient aucune bactérie cultivable sur gélose nutritive non sélective. Les essais ont ensuite consisté à introduire à intervalles de temps réguliers dans la carotte de sol, un volume d'eaux usées domestiques aux caractéristiques chimiques et bactériologiques déterminées, à recueillir les eaux qui ont percolé, et à déterminer également les caractéristiques chimiques et bactériologiques. Ces eaux usées ont ainsi été introduites aux différentes charges de 50, 100 et $250 \mathrm{~mL}$.

Pour l'application de chaque charge, un volume d'eau de la charge considérée est versé toutes les 30 minutes au-dessus de la colonne de sol. Par exemple, pour l'application de la charge de $50 \mathrm{~mL}$, un volume de $50 \mathrm{~mL}$ d'eau usée est introduit toutes les 30 minutes au-dessus de la colonne de sol en carotte. En se servant des résultats des travaux préliminaires, la fréquence de 30 minutes a été choisie dans le double but d'éviter un déséchage de la surface du dessus de la colonne de sol, et d'éviter aussi une perfusion d'eau usée en continu.

Les trois charges d'eau ont été appliquées sur la même carotte de sol. Entre l'application de la charge $50 \mathrm{~mL}$ et celle de $100 \mathrm{~mL}$ d'eau, de même qu'entre l'application de cette dernière et celle de $250 \mathrm{~mL}$ d'eau usée, de l'eau distillée stérilisée à l'autoclave a été introduite en continu au-dessus de la colonne de sol de façon à maintenir presque constant un volume de 10 litres d'eau au-dessus de cette colonne de sol. Cette introduction d'eau distillée stérilisée a duré chaque fois 15 jours et a visé à débarrasser la colonne de sol des cellules bactériennes provenant de l'introduction d'eau usée précédente. Juste après ce traitement, la colonne de sol est laissée pendant 30 jours dans le but de la sécher, avant le début de l'application de la charge suivante d'eau usée.

Pour chaque charge d'eau appliquée, les eaux introduites dans la colonne du sol proviennent chaque fois des eaux usées domestiques fraîchement recueillies d'un quartier populeux de Yaoundé. Les eaux qui ont percolé ont été recueillies par intervalles de temps réguliers, le premier intervalle étant considéré à partir de l'instant d'obtention de la première goutte d'eau ayant percolé. Le temps de collecte des gouttes d'eau percolée a été divisé en plusieurs intervalles dans le but de déterminer les variations temporelles des volumes d'eau percolée, ainsi que les fluctuations temporelles des caractéristiques des eaux qui percolent. Six intervalles de temps ont ainsi été choisis de manière à obtenir un volume de percolat qui puisse permettre la réalisation des analyses. Les intervalles de temps ont ainsi varié d'une charge d'eau à l'autre (la durée totale du temps de collecte des gouttes d'eau percolée varie d'une charge d'eau à l'autre). Pour chaque charge d'eau appliquée, les analyses ont été effectuées sur six percolats recueillis. Ces essais ont été réalisés à la température du laboratoire.

Les paramètres microbiologiques considérés ont été les coliformes thermotolérants isolés sur le milieu Endo (Bio-Rad) et les streptocoques fécaux isolés sur le milieu gélose à Bile Esculine Azide (Bio-Rad), comme préconisé par MARCHAL et al. (1991). Ces deux groupes de microorganismes ont été isolés par la technique des membranes filtrantes (ROMPRE et al., 2002). Les deux groupes de bactéries ont été choisis en raison de leur importance dans l'évaluation de la qualité microbiologique des eaux de boisson, les eaux souterraines étant classées parmi les ressources hydriques les plus consommées dans bon nombre de régions du monde (HASLAY et LECLERC, 1993; OMS, 1997; VILAGINES, 2003). Les variables physico-chimiques prises en compte ont été le $\mathrm{pH}$, la conductivité électrique et l'azote ammoniacal $\left(\mathrm{NH}_{4}^{+}\right)$; leurs valeurs ont été mesurées par les techniques préconisées par RODIER (1996). Certains paramètres chimiques n'ont pu être analysés pour des raisons de logistiques.

\section{RÉSULTATS ET DISCUSSION}

Les eaux introduites dans la carotte de sol pour l'ensemble des trois charges appliquées sont légèrement basiques $(\mathrm{pH}$ variant de 7,43 à 8,02 ). Leurs conductivités électriques varient de 6320 à $6650 \mu \mathrm{S} / \mathrm{cm}$, et leurs concentrations en $\mathrm{NH}_{4}^{+}$de 43,75 à $50 \mathrm{mg} / \mathrm{L}$ (Tableau 1). Les concentrations des coliformes thermotolérants varient de $19 \times 10^{6}$ à $33 \times 10^{6}$ 
Tableau 1 Caractéristiques des eaux usées introduites dans la carotte de sol et par charge d'eau appliquée.

Table 1 Characteristics of the wastewater introduced into the soil column, for each applied water load.

\begin{tabular}{|l|c|c|c|}
\hline \multirow{2}{*}{ Paramètres analysés } & \multicolumn{3}{|c|}{ Charge d'eau appliquée } \\
\cline { 2 - 4 } & $50 \mathrm{~mL}$ & $100 \mathrm{~mL}$ & $250 \mathrm{~mL}$ \\
\hline $\mathrm{pH}$ & 7,92 & 8,02 & 7,43 \\
\hline Conductivité électrique $(\mu \mathrm{S} / \mathrm{cm})$ & 6650 & 6600 & 6320 \\
\hline $\mathrm{NH}_{4}^{+}(\mathrm{mg} / \mathrm{L})$ & 48 & 43,75 & 50 \\
\hline Coliformes thermotolérants $(\mathrm{UFC} / 100 \mathrm{~mL})$ & $19 \times 10^{6}$ & $25 \times 10^{6}$ & $33 \times 10^{6}$ \\
\hline Streptocoques fécaux $(\mathrm{UFC} / 100 \mathrm{~mL})$ & $4 \times 10^{6}$ & $21 \times 10^{6}$ & $18 \times 10^{6}$ \\
\hline
\end{tabular}

UFC/ $100 \mathrm{~mL}$, et celles des streptocoques fécaux de 4 x $10^{6}$ à $21 \times 10^{6} \mathrm{UFC} / 100 \mathrm{~mL}$ (Tableau 1).

Le temps qui sépare le début de l'introduction des eaux usées dans la colonne de sol et l'instant de l'écoulement de la première goutte d'eau percolée a été déterminé pour chaque charge d'eau appliquée. Ce temps était de 12 heures lorsque la charge de $50 \mathrm{~mL}$ d'eau a été appliquée. Il était de 10,58 heures et de 9,47 heures, respectivement lorsque les charges de $100 \mathrm{~mL}$ et de $250 \mathrm{~mL}$ d'eau ont été appliquées (Figure 3). Le temps de collecte des gouttes d'eau percolée à partir de l'instant de la première goutte d'eau percolée a été de 15 heures pour la charge de $50 \mathrm{~mL}$, de 17,77 heures pour la charge de $100 \mathrm{~mL}$, et de 33,81 heures pour la charge de $250 \mathrm{~mL}$ d'eau appliquée (Figure 3). Le temps nécessaire pour obtenir la première goutte d'eau percolée apparaît ainsi plus long à faible charge d'eau

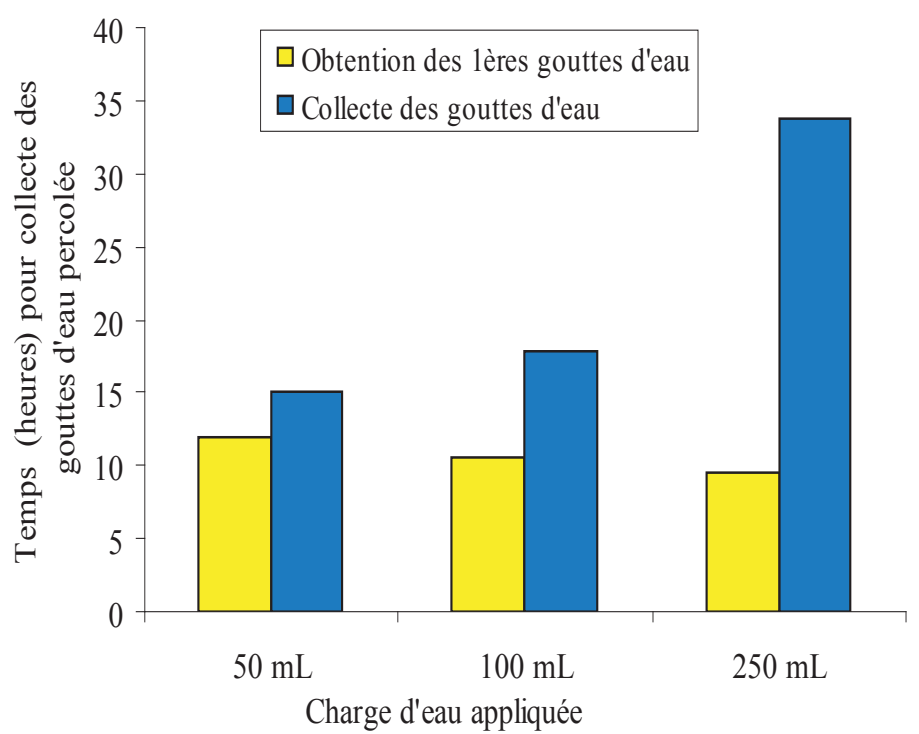

Figure 3 Temps nécessaire pour obtenir la première goutte d'eau percolée, et durée de collecte des gouttes d'eau percolée, par charge d'eau appliquée.

Duration time necessary to obtain the first drop of percolated water, and duration time for drops water collection, for each applied waterload. appliquée, et relativement plus court à charge d'eau appliquée élevée. Par contre, le temps nécessaire à la collecte d'un volume d'eau percolée adéquat pour effectuer les analyses est court à faible charge d'eau appliquée, et relativement plus long à charge d'eau relativement élevée, probablement à cause du colmatage des pores par les matières colloïdales et les matières grasses pouvant être contenues dans les eaux introduites.

Pour chaque charge, le temps de collecte des gouttes d'eau percolée a été divisé en six intervalles. Les volumes d'eau écoulés par intervalle de temps ont été mesurés, et le volume moyen d'eau percolée par heure et par intervalle de temps (I) a été calculé. L'intervalle I-1 correspond au premier intervalle, et l'intervalle I- 6 correspondant au $\sigma^{\mathrm{e}}$ intervalle de temps. En introduisant la charge de $50 \mathrm{~mL}$ d'eaux usées dans la colonne de sol, le volume d'eau écoulé par heure pendant les six intervalles de temps a varié de 20,33 mL à $29 \mathrm{~mL}$ (Figure 4). Ces volumes ont varié de 18,3 à $23 \mathrm{~mL} /$ heure pour la charge de $100 \mathrm{~mL}$ d'eaux usées, et de 14,6 à 21,5 mL/heure pour la charge de $250 \mathrm{~mL}$ (Figure 4). Le volume moyen d'eau écoulé par heure est ainsi élevé à faible charge, et relativement faible à charge d'eau appliquée élevée. Pour chacune des trois charges d'eau appliquées, les volumes des eaux écoulées sont faibles au début des percolations, et augmentent relativement dans le temps au fur et à mesure que le volume cumulé d'eaux usées introduites dans la colonne du sol est élevé (Figure 4).

Les analyses ont été effectuées sur les gouttes d'eau collectées au bout de chaque intervalle de temps. Six échantillons d'eau

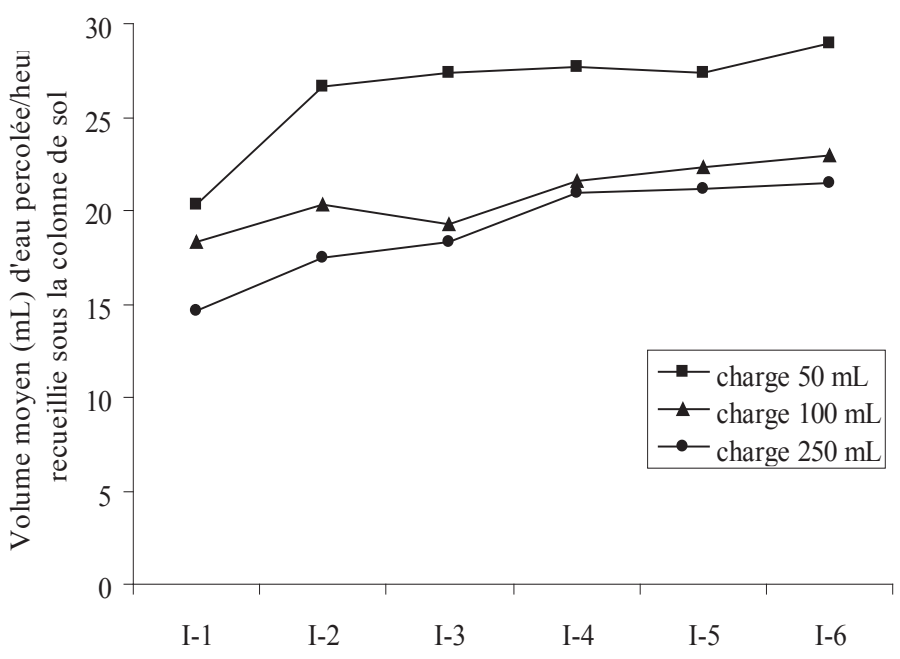

Intervalles de temps de collecte d'échantillons d'eau percolée sous la colonne de sol

Figure 4 Variation en fonction des six intervalles de temps d'analyse et des charges d'eau appliquées, des volumes moyens d'eau percolée par heure, recueillie sous la carotte de sol.

Variation during the six periods of analysis and for each applied waterload, of averages of volumes of percolated water per hour, collected under the soil column. 
percolée pour analyse ont ainsi été obtenus pour chaque charge. Il ressort de ces analyses que les valeurs moyennes pour l'ensemble des charges d'eau varient de 5,70 à 7,32 pour le $\mathrm{pH}$, de $80 \mu \mathrm{S} / \mathrm{cm}$ à $112 \mu \mathrm{S} / \mathrm{cm}$ pour la conductivité électrique, et de $0,84 \mathrm{mg} / \mathrm{L}$ à $1,06 \mathrm{mg} / \mathrm{L}$ pour la concentration en $\mathrm{NH}_{4}^{+}$ (Tableau 2).

La valeur moyenne de la concentration des coliformes thermotolérants pour les six analyses a été de $7 \mathrm{UFC} / 100 \mathrm{~mL}$ d'eau percolée pour la charge de $50 \mathrm{~mL}$. Elle a été de $3294 \mathrm{UFC} / 100 \mathrm{~mL}$ et de $5884 \mathrm{UFC} / 100 \mathrm{~mL}$, respectivement pour la charge de $100 \mathrm{~mL}$ et de $250 \mathrm{~mL}$ (Tableau 2). La concentration des streptocoques fécaux a été de 4 UFC/ $100 \mathrm{~mL}$ lorsque la charge de $50 \mathrm{~mL}$ d'eau a été appliquée, et de $145 \mathrm{UFC} / 100 \mathrm{~mL}$ lorsque la charge de $250 \mathrm{~mL}$ d'eau a été appliquée (Tableau 2). On note également une variation de la valeur de l'écart-type d'un groupe de bactéries à l'autre, et d'une charge d'eau à l'autre (Tableau 2).

Les caractéristiques chimiques de la colonne de sol étudiée sont présentées dans le tableau 3. Les deux horizons de sol ont un $\mathrm{pH}$ acide. La capacité de rétention en eau est de 43,62\% dans l'horizon $\mathrm{H}_{\mathrm{S}}$, et de 43,64\% dans l'horizon $\mathrm{H}_{\mathrm{I}}$ (Tableau 3). Les deux horizons de sol présentent la même concentration en phosphore, alors que les teneurs en carbone et en azote dans l'horizon $\mathrm{H}_{\mathrm{S}}$ sont relativement faibles par rapport à l'horizon $\mathrm{H}_{\mathrm{I}}$ (Tableau 3).

Les valeurs moyennes du $\mathrm{pH}$ des eaux percolées à travers les deux horizons de sol varient de 5,70 à 7,32 (Tableau 2), alors que les valeurs du $\mathrm{pH}$ des eaux introduites dans la colonne variaient de 7,43 à 8,02 (Tableau 1). Il apparaît ainsi évident que la nature du $\mathrm{pH}$ de ces deux horizons influence fortement celle des eaux infiltrées qui parviennent à la nappe phréatique. En rapprochant les caractéristiques des eaux introduites dans la colonne de sol (Tableau 1) de celles des eaux qui ont percolé (Tableau 2), on relève que les deux horizons du sol les plus proches du toit de la nappe phréatique retiennent les éléments ioniques des eaux d'infiltration, conduisant ainsi à une très forte diminution du degré de salinité des eaux souterraines.

Le rapport concentrations en éléments et bactéries de l'eau collectée (Tableau 2) sur concentrations en éléments et bactéries de l'eau introduite dans la colonne de sol (Tableau 1) varie de 0,76 à 0,92 pour le $\mathrm{pH}$, de 0,012 à 0,017 pour la conductivité électrique, de 0,017 à 0,024 pour l'azote ammoniacal, de $1,317 \times 10^{-4}$ à $3,68 \times 10^{-7}$ pour les coliformes thermotolérants, et de $2,73 \times 10^{-5}$ à $8,05 \times 10^{-6}$ pour les streptocoques fécaux. Il en résulte une réduction du nombre d'ions et du nombre de bactéries cultivables qui traversent la colonne de sol,

Tableau 2 Valeurs moyennes (et écart-type) des paramètres analysés au cours des six intervalles de temps, pour chaque charge d'eaux usées appliquée.

Table 2 Average values ( \pm standard deviation) of the parameters analyzed during the six time periods, for each applied water load.

\begin{tabular}{|l|c|c|c|}
\hline \multirow{2}{*}{ Paramètres analysés } & \multicolumn{3}{|c|}{ Charge d'eau appliquée } \\
\cline { 2 - 4 } & $50 \mathrm{~mL}$ & $100 \mathrm{~mL}$ & $250 \mathrm{~mL}$ \\
\hline $\mathrm{pH}$ & $7,32(0,09)$ & $6,10(0,06)$ & $5,70(0,36)$ \\
\hline Conductivité électrique $(\mu \mathrm{S} / \mathrm{cm})$ & $99,83(11,53)$ & $112(49,67)$ & $79,83(7,03)$ \\
\hline $\mathrm{NH}_{4}^{+}(\mathrm{mg} / \mathrm{L})$ & $0,84(0,12)$ & $1,06(0,26)$ & $0,99(0,09)$ \\
\hline $\begin{array}{l}\text { Coliformes thermotolérants } \\
(\mathrm{UFC} / 100 \mathrm{~mL})\end{array}$ & $7(4)$ & $3294(2019)$ & $5884(3819)$ \\
\hline Streptocoques fécaux $(\mathrm{UFC} / 100 \mathrm{~mL})$ & $4(3)$ & $574(448)$ & $145(183)$ \\
\hline
\end{tabular}

Tableau 3 Valeurs des paramètres chimiques analysés dans les deux horizons de sol formant la colonne de sol étudiée.

Table 3 Chemical parameter values for each of the two soil horizons that formed the studied soil column.

\begin{tabular}{|c|c|c|c|c|c|c|}
\hline \multirow{2}{*}{$\begin{array}{c}\text { Horizons de la } \\
\text { colonne de sol }\end{array}$} & \multicolumn{6}{|c|}{ Paramètres analysés } \\
\cline { 2 - 7 } & $\mathrm{pH}$ & CRE* $(\%)$ & Fe (mg/g) & $\mathrm{C}(\mathrm{mg} / \mathrm{g})$ & $\mathrm{N}(\mathrm{mg} / \mathrm{g})$ & $\mathrm{P}(\mathrm{mg} / \mathrm{g})$ \\
\hline $\mathrm{H}_{\mathrm{S}}$ & 4,43 & 43,62 & 0,75 & 4,10 & 0,10 & 26,10 \\
\hline $\mathrm{H}_{\mathrm{I}}$ & 4,56 & 43,64 & 0,70 & 4,30 & 0,20 & 26,10 \\
\hline
\end{tabular}

*CRE: Capacité de Rétention en Eau 
réduction imputable à leur rétention par le sol. À la charge de $50 \mathrm{~mL}$ d'eau appliquée, cette réduction a été de l'ordre de 7 unités logarithmiques pour les coliformes thermotolérants, et de 6 unités logarithmiques pour les streptocoques fécaux. En appliquant la charge de $250 \mathrm{~mL}$, la réduction a plutôt été de l'ordre de 6 unités logarithmiques pour les coliformes thermotolérants, et de 7 pour les streptocoques fécaux. La réduction de la concentration microbienne circulante observée dans les eaux percolées a été de l'ordre de 7 unités logarithmiques pour les deux groupes de bactéries avec la charge $100 \mathrm{~mL}$. À charge d'eau usée élevée, la colonne de sol semble ainsi retenir plus de streptocoques fécaux que de coliformes thermotolérants. Ce comportement de la colonne de sol apparaît inversé lorsque la charge d'eau appliquée est faible.

La présente étude relève l'importance de la charge d'eau appliquée sur la rétention des groupes de bactéries isolées. Les variations des volumes d'eau introduits au-dessus de la colonne de sol peuvent influencer la vitesse d'infiltration (de percolation) de l'eau dans la colonne de sol, ainsi que celle du transport des cellules bactériennes contenues dans ces eaux. Le flux advectif des bactéries transportées dans les eaux d'infiltration à travers les colonnes de sol a été mentionné comme lié à la force et à la vitesse d'écoulement de l'eau dans les pores de la colonne de sol (HEISE et GUST, 1999), ces dernières influençant les coefficients de filtration et les coefficients de dispersion hydrodynamique des microorganismes présents (MATTHESS et al., 1988; UNICE et LOGAN, 2000). Certaines études ont révélé sous conditions contrôlées de l'abondance des populations de Arthrobacter, Bacillus et Proteobacter dans le sol, une différence entre les faibles charges et les fortes charges d'eau contaminée appliquées au-dessus des colonnes de sol, différence attribuée à la différence de vitesse de transport des bactéries (BALKWILL et al., 1998). La mobilité de certaines souches bactériennes telles Pseudomonas stitzeri KC, $P$. fluorescens P17 et $P$. putida KT2442 a parfois été indiquée comme favorisant leur transport par l'intermédiaire des eaux d'infiltration dans le sol, mais sous faible vitesse d'infiltration du fluide (LOGAN, 2004).

Certains travaux réalisés en introduisant à fréquence régulière dans une colonne de sol insaturé et une colonne de sol saturé, une eau usée contenant les cellules E. coli, ont montré que le rendement épuratoire de la colonne de sol insaturé est élevé, la concentration des cellules E. coli dans les eaux qui ont percolé étant faible par rapport à celle des eaux qui ont percolé dans la colonne de sol saturé, bien que les deux types de sol aient les coefficients de filtration de premier ordre relativement proches (POWELSON et MILLS, 2001). La relative diminution des potentialités de rétention des cellules des bactéries coliformes thermotolérants, parallèlement à l'augmentation de la charge d'eau appliquée, serait liée à la perturbation par les mouvements de l'eau, des configurations des surfaces des particules de sol, et celles des cellules bactériennes et des molécules chimiques.

Cette étude a aussi montré qu'à charge d'eau appliquée relativement élevée, le nombre d'unités logarithmiques de réduction de l'abondance des coliformes thermotolérants est relativement faible par rapport à celui des streptocoques fécaux. D'autres études ont mentionné, dans l'évaluation de la qualité bactériologique des eaux souterraines en région équatoriale au Cameroun, des concentrations des coliformes fécaux et des coliformes thermotolérants supérieures à celles des streptocoques fécaux dans plusieurs de leurs sites d'études (NOLA et al., 1998, 2000, 2002). Cette prédominance numérique des bactéries coliformes fécaux par rapport aux streptocoques fécaux dans les eaux souterraines de cette région souvent caractérisée par une forte pluviométrie (SUCHEL, 1988), résulterait de la faible rétention des coliformes thermotolérants, tout au moins au niveau des deux horizons de sol du toit de la nappe phréatique.

L'écart-type sur la valeur de la concentration des bactéries présentes dans les eaux percolées (Tableau 2) montre que la rétention des microorganismes est un processus dynamique dans le temps. La rétention des microorganismes à la surface des particules du sol se produit par adhésion à ces surfaces, des macromolécules pariétales des bactéries (JUCKER et al., 1998). Son caractère dynamique peut résulter du caractère dynamique des propriétés physico-chimiques du milieu environnant (YEE et al., 2000). Les variations des propriétés des parois des bactéries, très souvent en réponse aux moindres modifications des conditions du milieu (SMETS et al., 1999; VAN DER MEI et al., 2000), peuvent aussi induire une variation de la rétention cellulaire observée.

La rétention des bactéries pourrait, dans un premier temps, être suivie de leur libération, puis cette dernière aussi suivie d'une possible re-rétention des cellules, traduisant une possible réversibilité de la rétention des microorganismes à travers la colonne de sol. Ces fluctuations indiquent la réversibilité $\mathrm{du}$ processus et traduisent le phénomène d'adsorptiondésorption-réadsorption cellulaire aux particules (CALLOW et FLETCHER, 1994). Cette réversibilité peut être causée par des interactions sous formes de signaux protéiques entre les cellules retenues (MILLSAP et al., 1998), ainsi que par l'activité cellulaire et les variations des propriétés chimiques des eaux qui percolent (MURPHY et GINN, 2000; VANCE, 2002). Elle se produit à des vitesses variant d'un horizon de sol à l'autre (HENDRY et al., 1999). La variabilité du coefficient de variation, c'est-à-dire la variation de la valeur de l'écart-type sur la concentration moyenne des microorganismes, d'abord d'un groupe de bactéries à l'autre, et ensuite d'une charge d'eau appliquée à l'autre, montre la variabilité de comportement de la 
colonne de sol en fonction des paramètres considérés. À partir des eaux de ruissellement en surface, les microorganismes pénètrent dans le sol, puis augmentent l'activité de leurs fonctions vitales, permettant une bonne migration en direction des eaux souterraines (GOUNOT, 1994). Cette migration est contrôlée par divers processus de transport tels l'advectiondispersion et l'adsorption-désorption, l'ensemble dépendant de la trajectoire de l'écoulement de l'eau (GOUNOT, 1994; MAYER et al., 1997).

Selon CALLOW et FLETCHER (1994), les mouvements des molécules d'eau perturbent la configuration des surfaces en contact, surface de la cellule bactérienne et surface de la particule de sol, surtout lorsque le processus de rétention est de longue durée. Il a aussi été noté que la percolation d'une eau usée introduite à régime variable dans une colonne de sol insaturé se produit avec une faible rétention des cellules Escherichia coli dans le sol (POWELSON et MILLS, 2001). Une bactérie qui s'infiltre dans le sol peut, par l'intermédiaire de ses polymères extracellulaires, augmenter significativement le transport des éléments chimiques en profondeur dans le sol, la mobilité de cette bactérie constituant un facteur favorisant le transport de ces éléments chimiques (LION et al., 2004).

Les fluctuations des valeurs des paramètres analysés dans les eaux percolées, pour chaque charge d'eau appliquée, pourraient traduire une relative réversibilité de la rétention des polluants dans la colonne de sol. Cette rétention évolue dans le temps à cause des modifications des propriétés des surfaces en contact, et dépend des conditions chimiques du milieu (RIJNAARTS et al., 1995; FOWLE et FEIN, 2000; YEE et al., 2000). Ces processus d'adsorption-désorption-réadsorption des cellules se produisent avec des vitesses qui varient avec la nature du sol en présence (HENDRY et al., 1999). Dans le temps et l'espace, le transport des bactéries dans un sol suppose un faible nombre de contacts (ou de rencontres) entre bactéries et particules de sol; ce transport est donc d'autant plus faible que la fréquence des contacts est important (LI et LOGAN, 1999).

Les variations des écarts-types des valeurs moyennes des variables analysées (Tableau 2) montrent que le degré de perturbation des surfaces solides et bactériennes/ou chimiques, en contact, varie avec la variation des volumes d'eau usée appliqués au-dessus de la colonne de sol. La qualité et l'abondance des cellules bactériennes dans les eaux du percolat ont parfois été mentionnées comme liées entre autres à la durée du temps de séjour des cellules dans le sol, à l'âge du sol et l'âge des cellules dans les eaux d'infiltration (RALFS et al., 2002). Il a aussi été noté que le nombre de cellules de Pseudomonas fluorescens dans certains percolats subit l'influence de leurs différents états physiologiques et métaboliques au cours de la percolation dans la colonne de sol, les cellules subissant souvent des modifications morphologiques durant le processus de migration (RALFS et al., 2002).
Certains auteurs ont mentionné que les éléments nutritifs, tels le carbone, l'azote et le phosphore dans le sol, peuvent stimuler une certaine activité bactérienne, perturber ainsi le processus de rétention cellulaire et induire sa réversibilité (MURPHY et GINN, 2000). Dans la présente étude, la colonne de sol étudiée est formée de deux horizons de sol $\mathrm{H}_{\mathrm{S}}$ et $\mathrm{H}_{\mathrm{I}}$ aux propriétés chimiques relativement différentes (Tableau $3)$.

En général, les conditions optimales de survie sont indispensables pour l'introduction effective de la bactérie dans le sol. Cette introduction est en général suivie d'une baisse momentanée de la biomasse de la bactérie introduite, suite à l'influence sur sa survie des facteurs abiotiques stables telles la texture et la granulométrie du sol, et des facteurs abiotiques dynamiques tels la température, la teneur en eau, le $\mathrm{pH}$ et les nutriments (VAN ELSAS et HEIJNEN, 1990).

Bien que la rétention des bactéries dans le sol soit en principe réversible (HENDRY et al., 1997), plusieurs auteurs, décrivant les variations de la concentration d'un microorganisme dans le sol, ont développé, en condition de carotte de sol, la théorie de filtration basée sur un modèle de rétention irréversible de $1^{\text {er }}$ ordre (SIMONI et al., 1998). Ce modèle tient compte de la concentration bactérienne initiale introduite dans le sol, de la concentration bactérienne mesurée à l'instant t dans le sol, de la porosité de la couche de sol, du rayon du tuyau collecteur, de la distance parcourue par l'eau d'infiltration, du facteur d'efficacité du collecteur, du coefficient de filtration et de l'efficacité de collision, cette efficacité de collision exprimant le rapport entre la concentration initiale des microorganismes et leur concentration instantanée le long de la colonne de sol (CAMESANO et LOGAN, 1998). La rétention des bactéries par le sol contribue à l'épuration des eaux usées qui s'infiltrent avec leurs contenus fécaux et non-fécaux, en direction de l'aquifère. Ce phénomène, pour SCHOLL et HARVEY (1992), survient lorsque l'association bactérie-surface de la particule de sol est largement contrôlée par les forces électrostatiques.

\section{CONCLUSION}

Cette étude montre que les deux horizons du sol les plus proches du toit de la nappe retiennent les éléments ioniques des eaux d'infiltration, conduisant à une très forte diminution du degré de salinité des eaux souterraines. La nature du $\mathrm{pH}$ de ces horizons du sol influence fortement celle des eaux infiltrées qui parviennent à l'eau souterraine. Le taux de rétention par ces horizons du sol, ou le taux de transfert à la nappe phréatique des bactério-contaminants des eaux d'infiltration, dépend, d'une part, du bactério-contaminant considéré et, d'autre part, de la charge d'eau polluée versée au-dessus de cette colonne de sol. Les caractéristiques des eaux qui percolent évoluent dans 
le temps, montrant que la rétention des polluants chimiques et bactériologiques des eaux d'infiltration par une colonne de sol est un processus dynamique.

\section{REMERCIEMENTS}

Ce travail a été réalisé avec l'appui de AIRE développement et celui du Fonds International de Coopération Universitaire - FICU (Agence Universitaire de la Francophonie).

\section{RÉFÉRENCES BIBLIOGRAPHIQUES}

BACHELIER G., 1959. Étude pédologique des sols de Yaoundé. Contribution à l'étude de la pédogenèse des sols ferralitiques. Agron. Trop., 14, 279-305.

BALKWILL D.L., MURPHY E.M., FAIR D.M., RINGELBERG D.B., WHITE D.C., 1998. Microbial communities in high and low recharge environments: implications for microbial transport in the vadose zone. Microb. Ecol., 35, 156-171.

CALLOW M.E., FLETCHER R.L., 1994. The influence of low surface-energy material on bioadhesion. - A review. Int. Biodeterior. Biodegrad., 34, 333-348.

CAMESANO T.A., LOGAN B.E., 1998. Influence of fluid velocity and cell concentration on the transport of motile and nonmotile bacteria in porous media. Environ. Sci. Technol., 32, 1699-1708.

CLAISSE G., 1968. Étude expérimentale de l'analyse aux trois acides. Comportement du quartz à l'attaque triacide. Cah. ORSTOM Ser. Pedol., 6, 129-149.

DEMOLON A., LEROUX D., 1952. Guide pour l'étude expérimentale du sol. Gauthier-Villars (Éditeur), Paris.

DZEDA B., KAISER M., MACH S., 1998. Bacteria and groundwater. Soil and Groundwater Pollution. Civil Engineering Dept., Virginia Tech. http://www.cee.vt.edu/ program-areas/environmental/teach/gwprimer/bacteria. html.

FENCHEL T., 2001. Microorganisms (microbes), role of. Encycl. Biodiversity, 4, 207-219.
FOWLE D.A., FEIN J.B., 2000. Experimental measurements of the reversibility of metal-bacteria adsorption reactions. Chem. Geol., 168, 27-36.

GOUNOT A.-M., 1994. Microbial ecology of groundwater. Dans : GIBERT J. \& STANFORD J. (Éditeurs), Groundwater Ecology. Academic Press, San Diego, 189219.

HASLAY C., LECLERC H., 1993. Microbiologie des eaux d'alimentation. Technique et Document, Lavoisier (Éditeur), Paris.

HEISE S., GUST G., 1999. Influence of the physiological status of bacteria on their transport into permeable sediments. Mar. Ecol. Progr. Ser, 190, 141-153.

HELENA B., PARDO R., VEGA M., BARRADO E., FERNANDEZ J.M., FERNANDEZ L., 2000. Temporal evolution of groundwater composition in an alluvial aquifer (Pisuerga river, Spain) by principal component analysis. Water Res., 34, 807-816.

HENDRY M.J., LAWRENCE J.R, MALOSZEWSKI P., 1997. Role of sorption in the transport of Klebsiella oxytoca though saturated silica sand. Ground Water, 35, 574-584.

HENDRY M.J., LAWRENCE J.R., MALOSZEWSKI P., 1999. Effects of velocity on the transport of two bacteria through saturated sand. Ground Water, 37, 103-112.

HOLT J.G., KRIEG N.R., SNEATH P.H.A., STALEY J.T., WILLIAMS S.T., 2000. Bergey's manual of determinative bacteriology. $9^{\text {th }}$ Ed., Lippincott W. \& Wilkins, Philadelphia.

HUMBEL F.X., 1969. Porosité, densité et perméabilité des sols ferralitiques rouges et jaunes près de Yaoundé. Cah. ORSTOM Ser. Sol, Yaoundé, 28 pages.

JUCKER B.A., ZEHNDER A.J.B., HARMS H., 1998. Quantification of polymer interactions in bacterial adhesion. Environ. Sci. Technol., 32, 2909-2915.

KORKKA-NIEMI K., LAIKARI H., 1994. Development of groundwater quality in Finnish wells in 1958-1991 based on geological and technological factors and human activities. Dans : SUOKKO T. \& SOVERI J. (Éditeurs), Future groundwater Resources at risk. Procc. Int. Conf., Helsinki, Finland, 13-16 june 1994, 139-159. 
LE JALLE C., 1998. Eau potable et assainissement dans les quartiers périurbains et les petits centres en Afrique. Gret (Editeurs), Paris.

LI Q., LOGAN B.E., 1999. Enhancing bacterial transport for bioaugmentation of aquifers using low ionic strength solution and surfactants. Water Res., 33, 1090-1100.

LION L.W., JENKINS M.B., DOHSE D.M., 2004. Microbial enhanced transport of hydrophobic organics in soil. http:// www.stormingmedia.us/62/6241/A62113.html.

LOGAN B.E., 2004. Bacterial transport in saturated, unsaturated and air-sparged porous media. http://www. niehs.gov/sbrp/home.html.

Macbeth Division of Kollmorgen Corporation, 1975. Determination of soil color-Munsel soil color charts. Macbeth Division of Kollmorgen Corporation (Éditeur), Baltimore, Maryland.

MADSEN E.L., GHIORSE W.C., 1993. Groundwater microbiology: subsurface ecosystem processes. Dans: Aquatic microbiology: an ecological approach. FORD T.E. (Éditeur), Blackwell Scientific Publications, Oxford, 167-213.

MARCHAL N., BOURDON J.L., RICHARD Cl., 1991. Les milieux de culture pour l'isolement et l'identification biochimique des bactéries, Doin (Éditeur), Paris.

MARTIN D., 1967. Géomorphologie des sols ferrallitiques dans le centre-Cameroun. Cah. ORSTOM Ser. Pedol., 5, 1989-2018.

MATTHESS G., PEKDEGER A., SCHROETER J., 1988. Persistence and transport of bacteria and viruses in groundwater - A conceptual evaluation. J. Contam. Hydrol., 2, 171-188.

MAYER A.S., CARRIÈRE P.P.E., GALLO C., PENNELL K.D., TAYLOR T.P., WILLIAMS G.A., ZHONG L., 1997. Groundwater quality. Water Environ. Res., 69, 778844.

MILLSAP K.W., VAN DER MEI H.C., BOS R., BUSSCHER H.J., 1998. Adhesive interaction between medically important yeasts and bacteria. FEMS Microbiol. Rev., 21, 321-336.

MULLER J.P., 1977. Microstructuration des structichrons rouges ferralitiques à l'amont des modelés convexes (centreCameroun). Aspect morphologique. Cah. ORSTOM Ser. Pedol., 11, 237-258.
MURPHY E.M., GINN T.R., 2000. Modelling microbial process in porous media. Hydrogeol. J., 8, 142-158.

NJINE T., MONKIEDJE A., NOLA M., SIKATI-FOKO V., 2001. Évaluation de la charge polluante et de la charge bactérienne des rejets des stations d'épuration à boues activées à Yaoundé (Cameroun). Cahiers Santé, 11, 79-84.

NJOPWOUO D., ORLIAC M., 1979. Note sur le comportement de certains minéraux à l'attaque triacide. Cah. ORSTOM Ser. Pedol., 17, 329-337.

NOLA M., NJINÉ T., BOUTIN C., 1998. Variabilité de la qualité des eaux souterraines dans quelques stations de Yaoundé (Cameroun). Mém. Biospeol., 25, 183-191.

NOLA M., NJINE T., DJUIKOM E., SIKATI FOKO V., 2000. Bacteria indicators dynamics in wells as influenced by well depth and well water column thickness, in Yaounde (Cameroon). Afr. J. Sci. Technol., 1, 82-91.

NOLA M., NJINE T., SIKATI F. V., DJUIKOM E., 2001. Distribution de Pseudomonas aeruginosa et Aeromonas hydrophila dans les eaux de la nappe phréatique superficielle en zone équatoriale au Cameroun et relations avec quelques paramètres chimiques du milieu. Rev. Sci. Eau, 14, 35-53.

NOLA M., NJINE, T., DJUIKOM, E., SIKATI FOKO, V., 2002. Faecal coliforms and faecal streptococci community in the underground water in an equatorial area in Cameroon (Central Africa): the importance of some environmental chemical factors. Water Res., 36, 3289-3297.

NOLA M., NJINE T., BOUTIN Cl., MESSOULI M., SERVAIS P., FOTO MENBOHAN S., NGO BIDJECK L.M., ZÉBAZÉ TOGOUET S.H., KEMKA N., 2004 a. Rétention de Escherichia coli d'une eau d'infiltration en sol équatorial au Cameroun (Afrique Centrale) : Rôle de divers horizons de sol. J. Cameroon Acad. Sci., 4, 107-116.

NOLA M., NJINET., BOUTIN Cl., SERVAIS P., MESSOULI M., ZEBAZE TOGOUET S.H., KEMKA N., NGO BIDJECK L.M., 2004b. Assessment of Streptococcus faecalis retention capacity by equatorial soil horizons in Cameroon (Central Africa). Bull. Soc. Hist. Nat. Toulouse, 140, 7-17.

OMS, 1997. Directives de qualité pour l'eau de boisson - Vol. 3. Surveillance et contrôle de l'approvisionnement en l'eau pour la communauté. OMS (Éditeur), Genève.

POWELSON D.K., MILLS A.L., 2001. Technical report waste management. J. Environ. Qual., 30, 238-245. 
RALFS C., KLAUTH P., USTOHAL P., KLUMPP E., 2002. Deposition and transport of metabolic active bacteria in soils: balance of biomass and influence of dividing behaviour. http://www.riob.org/divers/megev/eaumontagne-2002/pdf/trevisan.pdf.

RIJNAARTS H.H.M., NORDE W., BOUWER E.J., LYKLEMA J., ZEHNDER A.J.B., 1995. Reversibility and mechanism of bacterial adhesion. Colloids Surf. B, 4, 522.

RODIER J., 1996. L'analyse de l'eau, Dunod (Éditeur), Paris.

ROMPRE A., SERVAIS P., BAUDART J., DE-ROUBIN M.R., LAURENT P., 2002. Detection and enumeration of coliforms in drinking water: current methods and emerging approaches. J. Microbiol. Methods, 49, 31-54.

SCHIJVEN J.F., HASSANIZADEH S.M., 2000. Removal of viruses by soil passage: overview of modelling, processes and parameters. Crit. Rev. Environ. Sci. Technol., 30, 49127.

SCHOLL M.A., HARVEY R.W., 1992. Laboratory investigations on the role of sediments surface and groundwater chemistry in transport of bacteria through a contaminated sandy aquifer. Environ. Sci. Technol., 26, $1410-1417$.

SERRANO S.E., 2001. Solute transport under non-linear sorption and decay. Water Res., 35,1525-1533.

SIMONI S.F., HARMS H., BOSMA T.N.P., ZEHNDER A.J.B., 1998. Population heterogeneity affects transport of bacteria through sand columns at low rates. Environ. Sci. Technol., 32, 2100-2105.

SMETS B.F., GRASSO D., ENGWALL M.A., MACHINIST B.J., 1999. Surface physicochemical properties of Pseudomonas fluorescens and impact on adhesion and transport through porous media. Colloids Surf. B, 14, 121139.

SOIL AND PLANT ANALYSIS COUNCIL, 1999. Soil Analysis Handbook of Reference Methods. CRC (Éditeur), New York.

STEVENS T.O., 1997. Subsurface microbiology and the evolution of the biosphere. Dans: The microbiology of the terrestrial deep subsurface. AMY P.S. \& HALDEMAN D.L. (Éditeurs), Lewis Publishers, Boca Raton, 205-223.
SUCHEL F.G., 1988. Les régions climatiques du Cameroun : Les climats du Cameroun. Thèse de Doctorat d'État, Université St-Etienne, France, 780 pages.

TEUTSCHG.,HERBOLD-PASCHKEK.,TOUGIANIDOU D., HAHN T., BOTZENHART K., 1991. Transport of microorganisms in the underground-processes, experiments and simulation models. Water Sci. Technol., 24, 309-314.

UNICE K.M., LOGAN B.E., 2000. Insignificant role of hydrodynamic dispersion on bacterial transport. J. Environ. Eng., 126, 491-500.

VAN DER MEI H.C., DE VRIES J., BUSSCHER H.J., 2000. X-ray photoelectron spectroscopy for the study of microbial cell surfaces. Surf. Sci. Rep., 39, 1-24.

VANCE D.B., 2002. Particulate transport in groundwater part II - Bacteria. http://2the4.net/paartbact.html.

VAN ELSAS J.D., HEIJNEN C.E., 1990. Methods for the introduction of bacteria into soil. Biol. Fertil. Soils, 10, 127-133.

VILAGINES R., 2003. Eau, environnement et santé publique. Lavoisier (Éditeur), Paris.

YEE N., FEIN J.B., DAUGHNEY C.J., 2000. Experimental study of the $\mathrm{pH}$, ionic strength, and reversibility behavior of bacteria-mineral adsorption. Geochim. Cosmochim. Acta, 64, 609-617.

YONGUE-FOUATEU R., 1986. Contribution à l'étude pétrographique de l'altération et des faciès de cuirassement ferrugineux des gneiss migmatitiques de la région de Yaoundé. Thèse Doctorat, Université de Yaoundé, 214 pages. 